IMPORTANCE OF FULL COULOMB INTERACTIONS FOR UNDERSTANDING THE ELECTRONIC STRUCTURE OF DELTA-PU

E. Gorelov, J. Kolorenc, T. Wehling, H. Hafermann, A. I. Lichtenstein, A. B. Shick, A. N. Rubtsov, M. I. Katsnelson, A. Landa, A. K. McMahan

April 2, 2010

Physical Review B 
This document was prepared as an account of work sponsored by an agency of the United States government. Neither the United States government nor Lawrence Livermore National Security, LLC, nor any of their employees makes any warranty, expressed or implied, or assumes any legal liability or responsibility for the accuracy, completeness, or usefulness of any information, apparatus, product, or process disclosed, or represents that its use would not infringe privately owned rights. Reference herein to any specific commercial product, process, or service by trade name, trademark, manufacturer, or otherwise does not necessarily constitute or imply its endorsement, recommendation, or favoring by the United States government or Lawrence Livermore National Security, LLC. The views and opinions of authors expressed herein do not necessarily state or reflect those of the United States government or Lawrence Livermore National Security, LLC, and shall not be used for advertising or product endorsement purposes. 


\title{
Importance of full Coulomb interactions for understanding the electronic structure of $\delta-\mathrm{Pu}$
}

\author{
E. Gorelov ${ }^{1}$, J. Kolorenc ${ }^{1,2}$, T. Wehling ${ }^{1}$, H. Hafermann ${ }^{1}$, A. I. Lichtenstein ${ }^{1}$, \\ A. B. Shick ${ }^{2}$, A. N. Rubtsov ${ }^{3}$, M. I. Katsnelson ${ }^{4}$, A. Landa ${ }^{5}$, and A. K. McMahan ${ }^{5}$ \\ ${ }^{1}$ Institute of Theoretical Physics, University of Hamburg, 20355 Hamburg, Germany \\ ${ }^{2}$ Institute of Physics, Academy of Sciences of the Czech Republic, Na Slovance 2, 18221 \\ Praha 8, Czech Republic \\ ${ }^{3}$ Department of Physics, Moscow State University, 119992 Moscow, Russia \\ ${ }^{4}$ Institute for Molecules and Materials, Radboud University of Nijmegen, NL-6525 ED \\ Nijmegen, The Netherlands \\ ${ }^{5}$ Physical and Life Sciences Directorate, Lawrence Livermore National Laboratory, \\ Livermore, CA 94551, USA
}

The solid-state properties of most elements are now well understood on the basis of quantum physics - with few exceptions, notably the element number 94, plutonium. Plutonium has six crystalline phases at ambient pressure, some of which are separated by unusual phase transitions with large discontinuities in volume, exhibit negative thermal expansion coefficients, or form exotic low-symmetry structures ${ }^{1,2}$. The main challenge to explain these anomalous properties is that the characteristic ingredient of actinides, the $5 f$ electronic states, are in the cross-over regime between the localized and delocalized (itinerant) behaviour in $\mathrm{Pu}^{3,4}$. The early part of the actinide series with the $5 f$ states being itinerant, i.e. part of the metallic bond, culminates with Pu; starting with $\operatorname{Am}(Z=95)$, the $5 f$ states are localized, nonbonding, and resemble the $4 f$ states in lanthanides. Both itinerant and localized regimes are well covered by existing theories, but they cannot be simply interpolated due to the importance of dynamical electron-electron correlations ${ }^{5,6}$. Here we present accurate quantum Monte Carlo calculations achieving previously inaccessible resolution. Obtained results demonstrate that interplay of the full 


\section{Coulomb interaction vertex with spin-orbital coupling is crucial for understanding the experimentally observed spectral properties of plutonium near the Fermi level.}

In the last few years there has been intense research, both theoretical and experimental, aimed to understand properties of metallic plutonium, one of the most complicated elements in the Periodic Table ${ }^{1}$. At atmospheric pressure plutonium metal exhibits six crystal structures upon heating from room temperature to its melting point of $913 \mathrm{~K}^{2}$ The low-temperature monoclinic $\alpha$ phase is stable up to $395 \mathrm{~K}$, while the facecentered-cubic (fcc) $\delta$ phase is stable between $593-736 \mathrm{~K}$. The $\delta$-Pu phase, which received a significant interest in the metallurgical community because of its high ductility, is quite different from the brittle ground-state $\alpha$-Pu phase. For instance: (i) $\delta$-Pu has an atomic volume of about $25 \%$ greater than $\alpha-\mathrm{Pu}$; (ii) the thermal expansion of $\delta \mathrm{Pu}$ is small and negative whereas it is very large and positive for $\alpha-\mathrm{Pu}$; (iii) the electronic specific heat coefficient of $\delta$-Pu is substantially larger than in $\alpha$-Pu while the magnetic susceptibility stays almost unchanged. For all Pu-phases, local magnetic moments are absent ${ }^{7}$ but the Pauli-like magnetic susceptibility and resistivity are an order of magnitude larger than for simple metals. This unusual behavior is related to the unique position of $\mathrm{Pu}$ in the actinides series, which is at the boundary between early actinides with itinerant $5 f$ electrons and the late actinides with localized $5 f$ electrons ${ }^{3,4,5}$.

Strong correlations among electrons in Pu result in a failure to predict the equilibrium volume of non-magnetic $\delta$-Pu within conventional local density approximation (LDA) $)^{8}$. Naïve attempts to account for $f$-electron correlations within the $\mathrm{LDA}+\mathrm{U}$ technique result in a non-magnetic ground state close to $f^{6}$ configuration when the so-called "fully-localized" (FL) double counting together with the constrained spin polarization ${ }^{9}$ is used. The use of different form of the double counting, "around-themean-field" (AMF) $-\mathrm{LDA}+\mathrm{U},{ }^{10}$ results in "quenching" of the spin and orbital magnetic moments and shows an admixture of the $f^{5}$ and $f^{6}$ configurations. The $\delta$-Pu equilibrium 
volume is calculated $^{9,10}$ in good agreement with experiment. However, this static meanfield approach is not able to reproduce experimental photoemission spectra (PES) of Pu, where a high peak, attributed to $f$-states, is clearly observed in the vicinity of the Fermi level.

The LDA+U technique takes into account only the static part of the self-energy and that is not sufficient to correctly reproduce spectral properties of strongly correlated systems. The full frequency dependence of the self-energy requires improved methods such as dynamical mean field theory (DMFT) ${ }^{13,14}$ that enables treatment of band-like and atomic-like behavior of electrons on the same footing. The first application of DMFT to $\mathrm{Pu}$ was performed by Savrasov et al. ${ }^{5}$ who explained the origin of volume expansion between $\alpha$-Pu and $\delta$-Pu phases in terms of competition between Coulomb repulsion and kinetic energy. The authors assumed that $\mathrm{Pu}$ has a $5 f^{5}$ configuration with an open $f^{5 / 2}$ subshell resulting in a local magnetic moment that is further screened by valence electrons via Kondo mechanism. Although the double minimum on the energy vs. volume curve, derived in this paper, provided a possible explanation for the volume collapse that accompanies the $\alpha \rightarrow \delta$ transition in $\mathrm{Pu}$, the calculated density of states (DOS) did not include the realistic multiplet structure seen in photoemission experiments ${ }^{15}$. Taking into consideration that all DMFT calculations ${ }^{5}$ were actually performed only for the fcc lattice, more realistic interpretation of this double minimum, as an origin of the negative thermal expansion (Invar effect) in $\delta$-Pu, was presented later $^{16}$.

The non-magnetic configuration, suggested in the AMF-LDA+ U calculations by Shick et al. ${ }^{10}$, served as a "starting point" for several DMFT studies. Pourovskii et al. ${ }^{17}$ tried to correct the deficiency of LDA+U DOS by adding dynamical fluctuations by means of DMFT in conjunction with the fluctuating exchange $T$-matrix technique $(T$ matrix FLEX) ${ }^{18}$. A similar LDA+DMFT approach, but employing the Hubbard-I 
approximation (HIA) ${ }^{19}$ for the quantum impurity solver, was used by Shick et al. ${ }^{20}$ to reproduce the distinct three-peak structure experimentally observed on PES of $\delta \cdot \mathrm{Pu}^{15}$.

Later, Pourovskii et al. ${ }^{12}$ were able to reproduce the experimentally observed large increase of the electronic specific-heat coefficient of $\delta \mathrm{Pu}$ as compared to the $\alpha$-Pu phase. In this study the $T$-matrix FLEX method was also used for the quantum impurity solver. Shim et $a l .{ }^{21}$ employed a vertex corrected one-crossing approximation for the quantum impurity problem ${ }^{14}$ and obtained $5 f$ occupancy of $\sim 5.2$ in calculations which also properly included multiplet effects. They concluded that plutonium becomes nonmagnetic due to Kondo screening below $\sim 800 \mathrm{~K}$. Moreover, they also confirmed the suggestion ${ }^{10,20}$ that the fractional $5 f$ occupancy in $\delta$-Pu arises from a mixture of two different atomic configurations $\left(f^{5}\right.$ and $\left.f^{6}\right)$ in the ground state. Finally, Zhu et al. ${ }^{22}$ used both an exact quantum Monte Carlo (QMC) method within the Hirsch-Fye scheme ${ }^{23}$ (without multiplet effects) and the Hubbard-I approximation ${ }^{19}$ (with multiplet effects) for the quantum impurity solver. They demonstrated sensitivity of the spectral properties of $\mathrm{Pu}$ to the $5 f$ occupancy and suggested on the basis of comparison to experimental data that the $5 f^{5}$ "open shell" Pu configuration was more likely than the "closed shell" $5 f^{6}$ configuration. Nevertheless, according to Refs. ${ }^{20-22}$, the three-peak multiplet structure observed in the DOS of bulk $\delta$-Pu metal is induced by non-vanishing admixture of atomic $f^{6}$ configurations into the ground state.

Quantum Monte Carlo methods represent an exact tool to solve the quantum impurity problem. There are, however, several issues to be addressed when these methods are applied to systems like $\delta$-Pu. The often employed auxiliary-field Hirsch-Fye QMC algorithm is computationally expensive for multiorbital calculations at low temperature, ${ }^{14}$ since the required computer time scales as $N_{\text {orb }}{ }^{2} / T^{3}$, where $N_{\text {orb }}$ is the number of orbitals on the impurity and $T$ is the temperature. Even more importantly, the Hirsch-Fye algorithm has an inherent limitation on the form of the electron-electron 
interaction that can be included in the Hamiltonian. Out of the full Coulomb interaction vertex

$$
\begin{aligned}
& \mathrm{H}_{\mathrm{U}}=\frac{1}{2} \sum_{\mathrm{ij \sigma} \sigma^{\prime}} \mathrm{U}_{\mathrm{ijkl}} \mathrm{c}_{\mathrm{i} \sigma}^{+} \mathrm{c}_{\mathrm{j} \sigma^{+}}, \mathrm{c}_{\mathrm{l} \sigma^{\prime}} \mathrm{c}_{\mathrm{k} \sigma} \\
& \mathrm{U}_{\mathrm{ijkl}}=\langle\mathrm{ij}|\mathrm{U}| \mathrm{kl}\rangle
\end{aligned}
$$

only the density-density contributions

$$
\begin{aligned}
& \mathrm{H}_{\mathrm{U}}=\frac{1}{2} \sum_{\mathrm{ij} \sigma}\left[\mathrm{U}_{\mathrm{ij}} \mathrm{n}_{\mathrm{i} \sigma} \mathrm{n}_{\mathrm{j}-\sigma}+\left(\mathrm{U}_{\mathrm{ij}}-\mathrm{J}_{\mathrm{ij}}\right) \mathrm{n}_{\mathrm{i} \sigma} \mathrm{n}_{\mathrm{j} \sigma}\right] \\
& \mathrm{U}_{\mathrm{ij}}=\langle\mathrm{ij}|\mathrm{U}| \mathrm{ij}\rangle \\
& \mathrm{J}_{\mathrm{ij}}=\langle\mathrm{ij}|\mathrm{U}| \mathrm{ji}\rangle
\end{aligned}
$$

can be treated with this method. In the case of the $\mathrm{Pu} f$-shell, for instance, the full vertex has 377 non-equivalent terms and only 91 of them have the diagonal density-density form. The remaining non-diagonal part consists of 42 spin-flip terms and 244 integrals of the most general four-index form. These non-diagonal contributions can reduce the effects of large spin-orbital splitting in relativistic density functional calculations of $\delta$-Pu and produce a multiplet structure near the Fermi level. Notably, T-matrix FLEX, Hubbard-I and one crossing approximations used in Refs. ${ }^{12,20-22}$ examine the full $f-f$ Coulomb interaction and its consequent term structure, so that the Hund's rule exchange is included in these models.

Recently, a new class of continuous-time QMC (CT-QMC) impurity solvers has been developed $^{24-26}$. These diagrammatic QMC approaches rely on a perturbation expansion of the partition function into Feynman diagrams and on re-summation of these diagrams into determinants. The weak coupling method ${ }^{24}$ uses a perturbation expansion in the interaction part of the Hamiltonian and its determinantal formulation originates in the Wick's theorem. The strong-coupling or hybridization expansion method ${ }^{25,26}$ treats the local interactions exactly and expands in the impurity-bath hybridization; the determinants emerge naturally from the trace over the bath states. Both weak and strong 
coupling CT-QMC methods can treat the full Coulomb interaction vertex and do not suffer from discretization problems inherent to the Hirsch-Fye QMC in the low temperature regime. Therefore, the CT-QMC formalism has an essential advantage compared to auxiliary-field methods when complex systems like $\delta$-Pu are considered. The application of hybridization expansion CT-QMC to the electronic structure of $\delta-\mathrm{Pu}^{27}$ shows an importance of complete rotationally invariant Coulomb interaction among $5 f$ electrons.

In this Letter we apply the weak coupling CT-QMC scheme to investigate effects of full Coulomb interaction on the electronic spectrum of $\delta$-Pu. We start from the relativistic LDA Green's function for $f$-electrons ${ }^{28}$, which is a $14 \times 14$ matrix. We take advantage of the rotational invariance of the Coulomb interaction and work in the natural relativistic basis of spherical harmonics in order to avoid the so-called three orbital terms like $c^{+}{ }_{i} c^{+}{ }_{j} c_{i} c_{k}$ that cause a large fermion sign problem in the weak-coupling CT-QMC scheme. In low-temperature simulations, we still encountered a sign problem related to odd numbers of small but negative non-diagonal Coulomb matrix elements. Since these processes were very rare (of the order of $1 \%$ of accepted QMC steps) we simply restricted the QMC process to even numbers of non-diagonal Coulomb terms of the general form $c^{+}{ }_{i} c^{+}{ }_{j} c_{l} c_{k}$. We parameterize the matrix $U_{i j k l}$ with the aid of four Slater integrals $F_{0}=4 \mathrm{eV}, F_{2}=8.34 \mathrm{eV}, F_{4}=5.57 \mathrm{eV}, F_{6}=4.12 \mathrm{eV}$ in concord with the general $\mathrm{LDA}+\mathrm{U}$ scheme $\mathrm{e}^{9,10}$. The numerical values correspond to the screened average Coulomb integral $U=4 \mathrm{eV}$ and the exchange interaction parameter $J=0.7 \mathrm{eV}$. The CT-QMC simulations are computationally very demanding due to the presence of the full Coulomb vertex, and we therefore perform only a single solution of the impurity problem with the LDA Green's function (the first iteration of the DMFT procedure). In order to increase accuracy of analytical continuation of the CT-QMC data to the real energy axis, we use a novel stochastic approach starting directly from quantities at Matsubara frequencies 
(measured in the course of the CT-QMC simulation) and not from the imaginary-time $\operatorname{axis}^{29}$

We check first the influence of spin-flip terms in the Coulomb vertex and compare the spectral function of $\delta$-Pu with results of diagonal-only Coulomb vertex for relatively high temperature of the order of $5000 \mathrm{~K}$ in Fig. 1. The main effect of the spinflip terms is related to much stronger interaction between the $j=5 / 2$ and $7 / 2$ subbands at the same energy, demonstrated as an increase of the $j=7 / 2$ signal around $-2 \mathrm{eV}$ where the $j=5 / 2$ component has a strong peak, and as an analogous enhancement of the $j=5 / 2$ signal around $1 \mathrm{eV}$. Broadening of the spectral function maxima due to multiplet splitting is observed as well.

The final result for the spectral function of $\delta$-Pu with the full Coulomb vertex at relatively low temperature of the order of $600 \mathrm{~K}$ is shown in Figs. 2 a,b. The position of the quasiparticle peak near the Fermi level as well as the spin-orbit splitting of the multiplet structure (Fig. 2b) in our CT-QMC simulations well reproduce the main features of the experimental photoemission spectra ${ }^{11,15}$. Note that a large contribution to the lower Hubbard bands (around $-1.7 \mathrm{eV}$ in the Fig. 2b) comes from the high energy $j=7 / 2$ states.

Since the CT-QMC data presented here are limited to the first iteration towards the complete DMFT solution, it is illustrative to make a comparison with the results of charge-density self-consistent LDA+HIA calculations. ${ }^{30}$ For bulk $\delta-\mathrm{Pu}$ (shown in Fig. 3) we find a reasonable semi-quantitative agreement between full-Coulomb vertex calculations within CT-QMC and HIA. There are two $j=5 / 2$ peaks, one close to the Fermi edge and the other at approximately $-1 \mathrm{eV}$ in HIA, which are consistent with two $j=5 / 2$ peaks shown in Fig. 2b. In addition, there is a peak around $-0.7 \mathrm{eV}$ of $j=7 / 2$ character in Fig. 3 that corresponds to the $j=7 / 2$ peak at $-0.2 \mathrm{eV}$ in CT-QMC (cf., Fig. 2b). Furthermore, an extra $j=7 / 2$ satellite peak around $-1.7 \mathrm{eV}$ is seen in both the HIA and CT- 
QMC calculations. The $j=7 / 2$ structure centered at about $0.5 \mathrm{eV}$ in the HIA originates from $f^{5} \rightarrow f^{6}$ transitions and lies completely above the Fermi edge with a tail slightly extending to negative energies only due to our use of a $0.06 \mathrm{eV}$ FWHM broadening function. The HIA spectral function above the Fermi level (not shown) continues to exhibit significant $j=5 / 2-7 / 2$ mixing analogous to Fig. 1 obtained with the CT-QMC method.

One may wonder if the $j=7 / 2$ satellite at $-1.7 \mathrm{eV}$ is related to the experimental peak at about the same energy in the Pu-surface PE spectrum. ${ }^{15}$ To clarify the comparison between the theory and experiment, we performed LDA+HIA calculations for a freestanding $\delta-\mathrm{Pu}$ monolayer. Resulting $j=5 / 2-7 / 2$ orbital resolved spectral function is shown in insert in Fig. 3. The $f$-states localization is clearly seen in the Pu-monolayer as the $f$ spectral weight is shifted away from the Fermi level, in particular the main $j=5 / 2$ peak is now located at $-1.6 \mathrm{eV}$. These calculations therefore suggest that the peak around $-1.6 \mathrm{eV}$ apparent in the experimental 1 ML PE spectrum (Fig. 2b, green curve) is of $j=5 / 2$ and not $j=7 / 2$ character.

In spite of quite reasonable agreement between CT-QMC and HIA, which supports the atomic-like origin of the observed PE three peak structure, one needs to keep in mind that the hybridization between $f$ - and conduction electrons is not accounted for very accurately in the Hubbard-I procedure. To examine possible effects of the hybridization more thoroughly, we studied a model consisting of a correlated $f$-shell explicitly coupled to several bath spin-orbitals that mimic the hybridization with conduction electrons. We used band-Lanczos algorithm ${ }^{31}$ to find the ground state as well as to calculate the oneparticle spectral function shown in Fig. 4. We were able to include up to 12 bath spinorbitals in addition to the 14 that represent the $f$-shell, at which point the corresponding many-body Hilbert spaces reached dimensions of the order of $10^{7}$. Arguably, such sparse discretization represents only a rather crude approximation of the actual environment the $\mathrm{Pu} f$-shell experiences in the real solid. Nevertheless, the solution of this model possesses 
several important properties. The ground state of the cluster $\{f$-shell + bath $\}$ is a singlet that correlates with the absence of local magnetic moments observed in plutonium. The corresponding $f$-shell occupation is fractional $(\sim 5.4)$ and even configurations with 4 and 7 $f$-electrons have appreciable weights. Despite this indication that the hybridization with the conduction electrons is relatively strong, the spectral features in the first $1-2 \mathrm{eV}$ below the Fermi level (Fig. 4) still strongly resemble the atomic $f^{6} \rightarrow f^{5}$ transitions characteristic for the Hubbard-I approximation (Fig. 3). This observation further justifies the interpretation of the photo-emission spectral features located near the Fermi edge as atomic-like multiplet transitions. Moreover, there is a large contribution of $j=7 / 2$ states below the Fermi level near $-1.5 \mathrm{eV}$ in a good agreement with our CT-QMC calculations that incorporate the full bath Green's function.

To conclude, we emphasize that the present calculations, using full rotationallyinvariant Coulomb interaction vertex, have been able reproduce the spectral properties of $\delta$-Pu in agreement with recent photoemission experiments. The large peak of $f$-character in the density of states near the Fermi level as well as multiplet-like structure with spinorbital splitting is obtained. The success of the CT-QMC calculation for a "simple" element further opens the way for its application to a host of fascinating compounds that challenge condensed-matter theorists.

\section{References}

1. Handbook on the Physics and Chemistry of the Actinides, ed. by Freeman, A.J. and Lander, G.H. (North-Holland, Amsterdam, 1984).

2. Hecker, S.S., Harbur, D.R., \& Zocco, T.G. Phase stability and phase transformations in Pu-Ga alloys. Prog. Materials Sci. 49, 429-485 (2004).

3. Johansson, B. Nature of $5 f$ electrons in the actinide series. Phys. Rev. B 11, 27402743 (1975). 
4. Katsnelson, M.I., Solovyev, I.V., \& Trefilov, A.V. $\alpha-\delta$ transition in plutonium as a Mott transition in an $f$ subsystem. JETP Lett. 56, 272-276 (1992).

5. Savrasov, S.Y., Kotliar, G., \& Abrahams, E. Correlated electrons in $\delta$-plutonium within a dynamical mean-field picture. Nature 410, 793-795 (2001).

6. Dai, X., Savrasov S.Y., Kotliar, G., Migliori A., Ledbetter, H., and Abrahams. E. Calculated phonon spectra of plutonium at high temperatures. Science 300, 953-955 (2003).

7. Lashley, J.C., Lawson, A., McQueenney, R.J. \& Lander, G.H. Absence of magnetic moments in plutonium. Phys. Rev. B 72, 054416 (2005).

8. Söderlind, P., Eriksson, O., Johansson, B., \& Wills, J. Structural properties of plutonium from first-principles theory. Phys. Rev. B 55, 1997-2004 (1997).

9. Shorikov, A.O., Lukoyanov, A.V., Korotin, M.A., \& Anisimov, V.I. Magnetic state and electronic structure of $\delta$ and $\alpha$ phases of metallic Pu and its compounds. Phys. Rev. B 72, 024458 (2005).

10. Shick, A.B., Drchal, V., \& Havela, L. Coulomb- $U$ and magnetic-moment collapse in $\delta$-Pu. Europhysics Letters 64, 588-594 (2005); F. Cricchio, F. Bultmark, and L. Nordström, Exchange energy dominated by large spin-currents in $\delta$-Pu, Phys. Rev. B 78, 100404(R) (2008).

11. Arko, A. J., Joyce, J. J., Morales, L., Wills J., Lashley, J., Wastin, F., Rebizant, J. Electronic structure of $\alpha$ - and $\delta$-Pu from photoelectron spectroscopy, Phys. Rev. $B$ 62, 1773-1779 (2000)

12. Pourovskii, L.V., Kotliar, G., Katsnelson, M.I., \& Lichtenstein, A.I. Dynamical mean-field theory investigation of specific heat and electronic structure of $\alpha$ - and S-Pu. Phys. Rev. B 75, 235107 (2007). 
13. Georges, A., Kotliar, G., Krauth, W., \& Rosenberg, M.J. Dynamical mean-field theory of strongly correlated fermion systems and the limit of infinite dimension. Rev. Mod. Phys. 68, 13-125 (1996).

14. Kotliar, G., Savrasov, S.Y., Haule, K., Udovenko, V.S., Parcollet, O., \& Marianetti, C.A. Electronic structure calculations with dynamical mean-field theory. Rev. Mod. Phys. 78, 865-951 (2006).

15. Havela, L., Gouder, T., Wastin, F., \& Rebizant, J. Photoelectron spectroscopy study of the $5 f$ localization in Pu. Phys. Rev. B 65, 235118 (2002).

16. Lawson, A.C., Roberts, J.A., Martinez, B., Ramos, M., Kotliar, G., Trouw, F.W., Fitzsimmons, M.R., Hehlen, M.P., Lashley, J.C., Ledbetter, H., McQueeney R.J., $\&$ Migliori, A. Invar model for $\delta$-phase Pu: thermal expansion, elastic and magnetic properties. Phil. Mag. 86, 2713-2733 (2006).

17. Pourovskii, L.V., Katsnelson, M.I., Lichtenstein, A.I., Havela, L., Gouder, T., Wastin, F., Shick, A.B., Drchal, V., \& Lander, G.H. Nature of non-magnetic strongly-correlated state in $\delta$-plutonium. Europhysics Letters 74, 479-485 (2006).

18. Katsnelson M.I., \& Lichtenstein, A.I. A local self-consistent perturbation scheme. Eur. Phys. J. B 30, 9-15 (2002).

19. Lichtenstein A.I., \& Katsnelson, M.I. Ab initio calculations of quasiparticle band structure in correlated systems: LDA++ approach, Phys. Rev. B 57, 6884-6895 (1998).

20. Shick, A., Kolorenč, J., Havela, L., Drchal, V., \& Gouder, T. Multiplet effects in the electronic structure of $\delta$-Pu, Am, and their compounds. Europhysics Letters 77, 17003 (2007).

21. Shim, J.H., Haule, K., \& Kotliar, G. Fluctuation valence in a correlated solid and the anomalous properties of $\delta$-plutonium. Nature 446, 513-516 (2007). 
22. Zhu, J.-X., McMahan, A.K., Jones, M.D., Durakiewicz, T., Joyce, J.J., Wills, J.M., $\&$ Albers, R.C. Spectral properties of $\delta$-plutonium: Sensitivity to $5 f$ occupancy. Phys. Rev. B 76, 245118 (2007).

23. Hirsch, J.E., \& Fye, R.M., Phys. Rev. Lett. 56, (1986) 2521.

24. Rubtsov, A.N., Savkin, V.V., \& Lichtenstein, A.I. Continuous-time quantum Monte Carlo method for fermions. Phys. Rev. B 72, 035122 (2005).

25. Werner, P., Comanac, A., de' Medici, L., Troyer, M., \& Millis, A.J.. Continuoustime solver for quantum impurity models. Phys. Rev. Lett. 97, 076405 (2006).

26. Werner P., \& Millis, A.J. Hybridization expansion impurity solver: General formulation and application to Kondo lattice and two-orbital models. Phys. Rev. B 74, 155107 (2006).

27. Marianetti, C.A., Haule, K., Kotliar, G., \& Fluss, M.F. Electronic coherence in $\delta$ Pu: A DMFT study. Phys. Rev. Lett. 101, 056403 (2008).

28. Anisimov, V.I., Aryasetiawan, F., \& Lichtenstein, A.I. First-principles calculations of the electronic structure and spectra of strongly correlated systems: the LDA+ $U$ method. J. Phys.: Condens. Matter. 9, 767-808 (1997).

29. Mishchenko, A.S., Prokof'ev N.V., Sakamoto A., \& Svistunov B.V. Diagrammatic quantum Monte Carlo study of Fröhlich polaron. Phys. Rev. B 62, 6317-6336 (2000).

30. Shick, A. B., Kolorenc, J., Lichtenstein, A. I., and Havela, L. Electronic structure and spectral properties of Am, Cm and Bk, Phys. Rev. B 80, 085106 (2009).

31. Meyer, H.-D., and Pal S. A band-Lanczos method for computing matrix elements of a resolvent, J. Chem. Phys. 91, 6195-6204 (1989).

Acknowledgements. This work performed under the auspices of the U.S. Department of Energy by Lawrence Livermore National Laboratory under Contract DE-AC52-07NA27344. Computer time was 
awarded for the Atlas Grand Challenge Proposal "Predictive Properties of Plutonium with Dynamical Mean Field Theory" at LLNL. A.L. would like to acknowledge the DFG Grant SFB668-A3. J.K. acknowledges financial support by Alexander von Humboldt foundation.

Competing interests statement. The authors declare that they have no competing financial interests.

Correspondence and requests for materials should be addressed to A. Lichtenstein (alichten@physnet.uni-hamburg.de)

Figure captions:

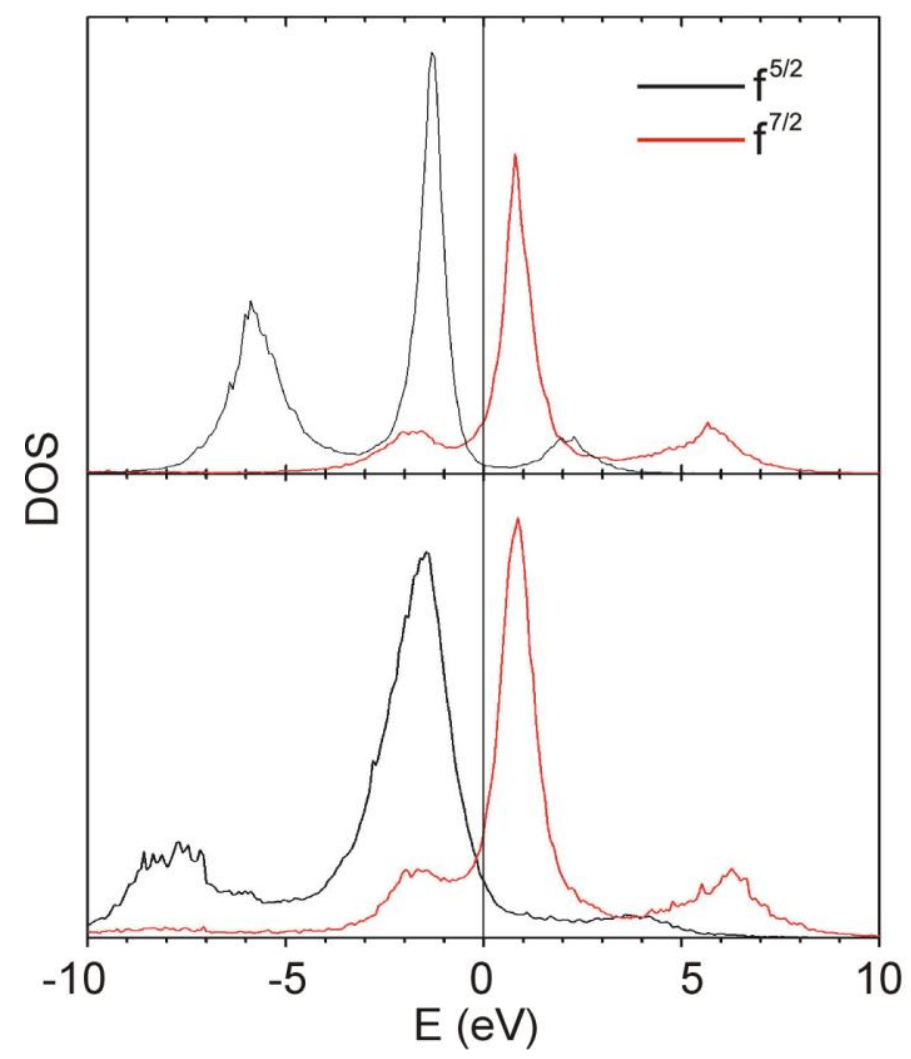

Fig. 1: Orbital resolved spectral function of Pu for high temperature with non-diagonal spin-flip Coulomb vertex (lower panel) and diagonal density-density interaction (upper panel). 


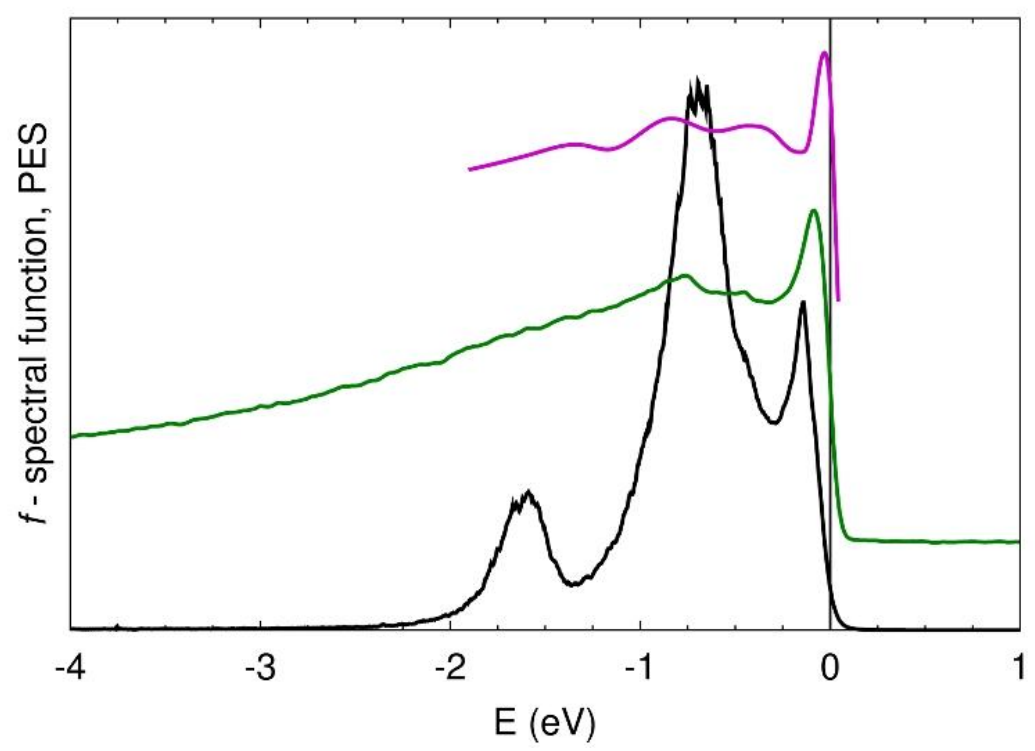

Fig. 2a: The $f$-electron spectral function of $\delta$-Pu calculated with the full Coulomb vertex at the temperature of $600 \mathrm{~K}$ (black) compared to two measurements of bulk photoemission spectra, Ref. [15] (violet) and Ref. [11] (green).

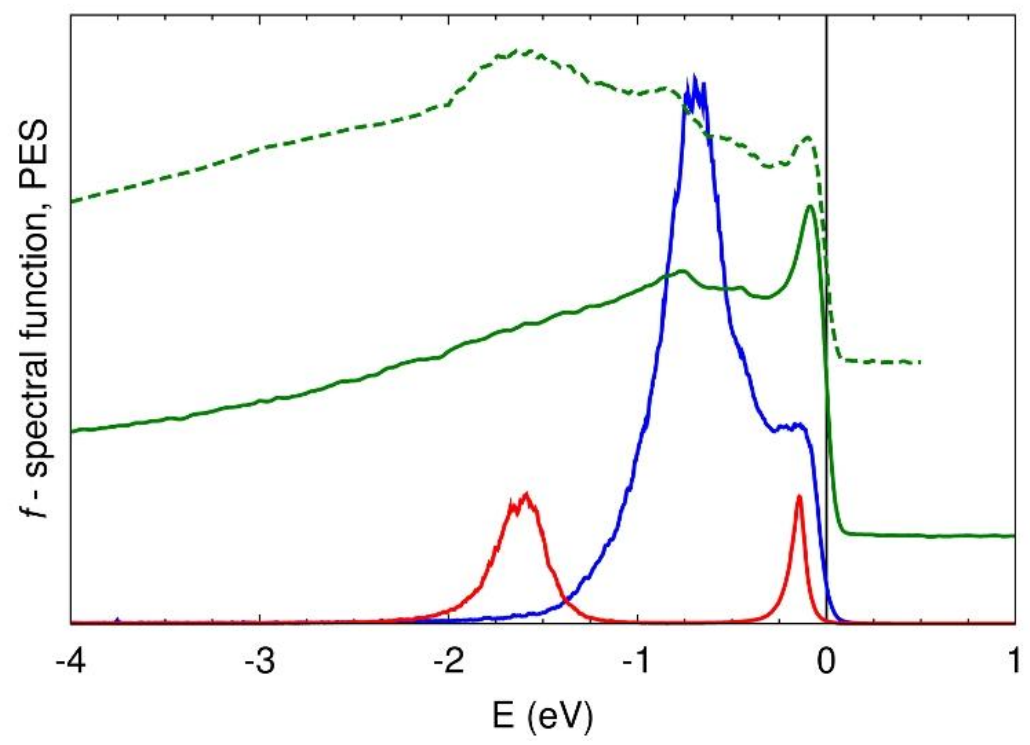

Fig. 2b: Orbital resolved $f$-electron spectral function from Fig. 2a ( $j=5 / 2$ contribution blue, $j=7 / 2$ component red) in comparison with experimental photoemission spectra of bulk (green, solid line) and surface (green, dashed line) of $\delta \mathrm{Pu}$ [15]. 


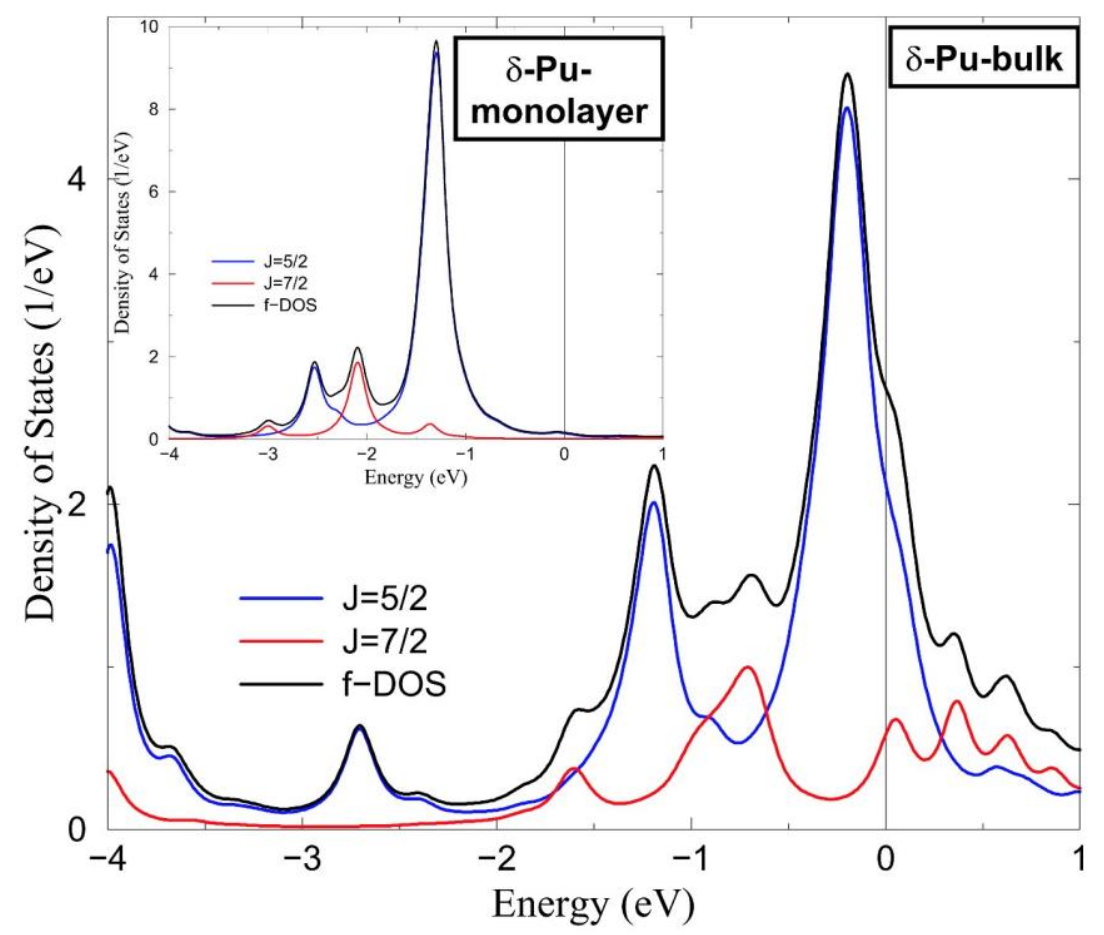

Fig. 3: Orbital resolved spectral function of Pu in the Hubbard-I approximation. The full Coulomb interaction causes strong admixture of high-energy $j=7 / 2$ and low-energy $j=5 / 2$ states. Insert shows the total and orbital resolved spectral function of the Pu-monolayer .

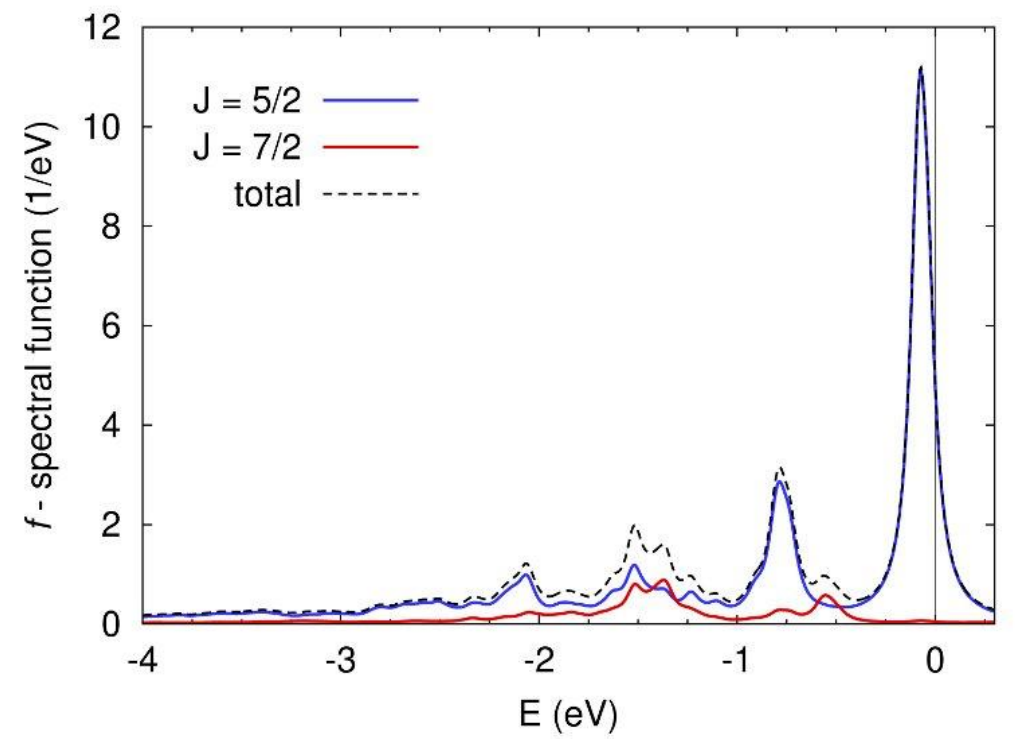

Fig. 4: Ground-state $(T=0 \mathrm{~K})$ spectral function of a $\mathrm{Pu} f$-shell coupled to 12 spin-orbitals representing the hybridization function. 